\title{
PERFIL EPIDEMIOLÓGICO DE POLICIAIS MILITARES DE UM GRANDE COMANDO DE POLICIAMENTO DO INTERIOR
}

\section{EPIDEMIOLOGIC PROFILE OF A GREAT MILITARY POLICE COMMAND}

\author{
Edivaldo Ferreira dos Santos ${ }^{1}$ \\ Orlando Soares Gomes Neto ${ }^{2}$ \\ Emilia Angela Lo Schiavo Arisawa ${ }^{3}$ \\ Alessandra Conceição da Silva ${ }^{4}$
}

Resumo: A profissão de policial militar (PM) está no topo daquelas suscetíveis a doenças físicas e psíquicas, pela exposição contínua em seu exercício a riscos psíquicos e físicos, com queda acentuada da qualidade de vida e riscos à saúde da função. Este estudo objetivou realizar retrato da prevalência de doenças que acometeram os policiais militares de um grande batalhão da Polícia Militar, entre Janeiro a Dezembro de 2014. A pesquisa é descritiva, quantitativa e retrospectiva, analisando dados estatísticos compilados a partir do banco de dados da Unidade do Comando de Policiamento do Interior Um, no período citado. Compuseram a amostra militares de três Batalhões que apresentam, aproximadamente, 1050 integrantes. Os dados, fornecidos pela Unidade Integrada de Saúde (UIS), basearam-se em levantamento interno, enviados em planilha mensal que discrimina doenças prevalentes em cada batalhão estudado. Os dados foram compilados de acordo com a Unidade Operacional, permitindo análises de cada batalhão e a comparativa entre os mesmos. Todos os dados foram submetidos à análise estatística descritiva, utilizando as medidas de tendência central (média, mediana e desvio padrão). As análises se concentraram nas doenças ortopédicas, psiquiátricas, cardiorrespiratórias, gastrointestinais, dermatológicas e renais que acometeram os PMs, no ano de 2014. Observou-se que as doenças ortopédicas prevaleceram nos três batalhões analisados (45\%), seguidas de doenças psiquiátricas (16\%) e doenças cardiorrespiratórias (16\%). Os resultados permitiram realizar um retrato da prevalência de doenças que acometeram os policiais militares de um batalhão, no período de Janeiro a Dezembro de 2014, observando-se as doenças ortopédicas como prevalentes.

Palavras-chave: Policial Militar; epidemiologia; riscos.

Abstract: Military policemen (MP) are on top of the professions susceptible to physical and mental illness, because they are continuously exposed to psychological and physical risks, with a marked decrease in quality of life and health risks. This study aimed to carry out a picture of the prevalence of diseases that attacked the military police battalion from January to December 2014. It is a descriptive, quantitative and retrospective study analyzing statistical data compiled from the database Policing the Interior Command Unit $A$, in the given period above. The sample was composed of three military battalions that have about 1,050 members. The data, provided by the Unit Integrated Health (UIS), were based on internal research, sent in monthly spreadsheets that discriminate against prevalent diseases in each battalion studied. The data were compiled on an individual basis, ie according to the operating unit, enabling the analysis of each battalion and comparative there between. All data were submitted to descriptive statistical analysis, using

\footnotetext{
${ }^{1}$ Graduado em Fisioterapia - Faculdade Anhanguera de Taubaté-SP. Especialista em Fisioterapia TraumatoOrtopédica com ênfase em Terapia Manual e Postural - Faculdade Anhanguera de Taubaté-SP. Mestrando em Bioengenharia - Universidade do Vale do Paraíba - UNIVAP, Brasil. E-mail: edivaldocop@bol.com.br.

${ }_{2}^{2}$ Graduado em Fisioterapia - Faculdade Diferencial - FACID/DEVRY. Educador físico - Universidade Federal do Piauí - UFPI. Mestrando em Bioengenharia - Universidade do Vale do Paraíba - UNIVAP. E-mail: landogneto@hotmail.com.

${ }^{3}$ Graduada em Ciências Biológicas, Modalidade Médica, pela Universidade de São Paulo - USP. Mestre em Farmacologia pela mesma Instituição. Doutora em Biopatologia Bucal pela Universidade Estadual Paulista Júlio de Mesquita Filho - UNESP (Campus São José dos Campos). Diretora da Faculdade de Ciências da Saúde (FCS) - Universidade do Vale do Paraíba - UNIVAP, Brasil. E-mail: mirela@univap.br.

${ }^{4}$ Graduada em Enfermagem pela Universidade Estácio de Sá. Mestranda em Bioengenharia - Universidade do Vale do Paraíba - UNIVAP, Brasil. Email: alessandra.conc@ig.com.br.
} 
the measures of central tendency (mean, median and standard deviation). The analysis focused on the orthopedic, psychiatric, cardiorespiratory, gastrointestinal, skin and kidney diseases which affected the military police in 2014. It was observed that orthopedic diseases prevailed in the three battalion analyzed (45\%), followed by psychiatric disorders (16\%) and cardiovascular diseases (16\%). The results allowed to carry a picture of the prevalence of diseases that affected the military police battalion in the period January to December 2014, observing that orthopedic diseases were prevalent.

Keywords: Police Military; epidemiology; risks.

\section{INTRODUÇÃO}

Conceitua-se "Qualidade de Vida" como uma concepção intrínseca ao indivíduo, relacionada ao seu posicionamento na vida, seus valores sociais e culturais, expectativas e perspectivas profissionais, objetivos e metas pessoais. É um fenômeno de percepção pessoal, de como sentir sua saúde e outros estados associados, como bem-estar e ocupação (SILVA et al., 2014). A atividade laboral é um dos fatores que interfere, significativamente, na qualidade de vida do indivíduo, atingindo, pontualmente, a saúde. Suas consequências variam entre os níveis de desenvolvimento, visto que em países menos desenvolvidos, com deficiência em saneamento básico, doenças relacionadas à fome e desigualdade social, predominam doenças transmissíveis. Por outro lado, em países mais desenvolvidos predominam doenças não transmissíveis (SILVA; SCHICHTING, 2012). As condições atuais de mudança de estilo de vida, com pouco tempo para atividade física, lazer e família, associado a um aumento substancial da violência e da criminalidade geram, consequentemente, o aumento de doenças não transmissíveis (FERREIRA; BONFIM; AUGUSTO, 2011).

Dentre as profissões existentes, a do policial militar está no topo daquelas suscetíveis a doenças não transmissíveis. Pelo exercício da função, estão, continuadamente, expostos a riscos psíquicos e físicos, com queda acentuada da qualidade de vida e riscos à saúde, pela assumpção rotineira da função de intermediador em situações de problemas humanos, de muitos conflitos e tensões (SILVA et al., 2014).

O Policial Militar "condiciona-se", no seu cotidiano, com a violência, a brutalidade e o risco de morte, bem como à elevada carga de trabalho e ao sofrimento. Destacase de outras categorias profissionais, por internalizar um nível elevado de estresse, gerando, prematuramente, desgaste físico e mental (GONÇALVES; VEIGA, RODRIGUES, 2012). O desgaste físico é gerado pelo acompanhamento a pé ou motorizado; pelo confronto físico, que pode gerar traumatismos, ferimentos contusos por projéteis ou arma branca; por lesões osteomusculares e pelo estilo de vida contemporâneo, que, frequentemente, evolui para cardiopatias. Esses eventos afetam os policiais militares, interferindo, drasticamente, na qualidade de suas vidas (SILVA et al., 2014).

Simões (2003) afirmou que o policial militar, na atividade de policiamento ostensivo, traz consigo equipamentos de proteção individual (EPIs) indispensáveis 
para sua segurança, dentre os quais estão: cinturão preto, arma de porte, algemas, rádio $\mathrm{HT}$, cassetete, que, juntos, pesam em torno de $2,10 \mathrm{~kg}$, aos quais soma-se, ainda, o peso dos coletes balísticos que variam em torno de $1,6 \mathrm{Kg}$ a $2,6 \mathrm{Kg}$, para os homens, e $1,1 \mathrm{Kg}$ a $1,8 \mathrm{Kg}$ para as mulheres. Dependendo do condicionamento físico do policial, os EPIs podem limitar o bom desempenho de suas atividades, colaborando com o aparecimento de sinais de cansaço, fadiga e dores em, praticamente, todo o sistema osteomuscular.

Acrescenta-se, a esse quadro, a incompatibilidade função-salário, que faz com que esses colaboradores procurem atividades extras, em seus horários de descanso, nas Unidades credenciadas da Polícia Militar ou atividades extra corporação, os famosos "bicos"; dedicando pouco ou quase nenhum tempo à prática de exercícios, levando, assim, um estilo de vida sedentário (GONÇALVES; VEIGA; RODRIGUES, 2012).

Diante da relevância do tema, este estudo teve como objetivo realizar um retrato da prevalência de doenças que acometeram os policiais militares de um grande Comando de Policiamento do Interior do Estado de São Paulo, no período de Janeiro a Dezembro de 2014.

\section{METODOLOGIA}

Este estudo foi iniciado após aprovação pelo Comitê de Ética em Pesquisa da Universidade do Vale do Paraíba ( $n^{\circ}$ CAAE 48685815.1.0000.5503). Os dados foram disponibilizados pelo Comandante do Policiamento do Interior Um, conforme Termo de Consentimento assinado, no qual constava a liberação das informações pela Unidade Integrada de Saúde (UIS), local onde eles se apresentam quando necessitam de atendimento médico ou outro suporte de competência da UIS. É um estudo descritivo, quantitativo e retrospectivo, com análise dos dados estatísticos compilados a partir do banco de dados da UIS, do referido Comando de Policiamento, referentes ao período de Janeiro a Dezembro de 2014.

A amostra foi constituída por efetivo composto por, aproximadamente, 1050 policiais militares de 03 (três) Unidades do Comando de Policiamento estudado, que contemplava o $1^{\circ}$ Batalhão de Polícia Militar do Interior - São José dos Campos/SP, o $41^{\circ}$ Batalhão de Polícia Militar do Interior - Jacareí/SP e o $46^{\circ}$ Batalhão de Polícia Militar do Interior - São José dos Campos/SP. A seleção dessas unidades decorre de sua importância na região e no estado de São Paulo.

Os dados utilizados no presente estudo foram fornecidos pela Unidade Integrada de Saúde, baseados em estatística realizada internamente pela sua chefia. Os diagnósticos foram realizados pelo médico responsável, que os agrupou, de acordo 
com o Código Internacional de Doenças (CID), em planilha discriminando as doenças prevalentes em cada mês, em cada batalhão que compôs a amostra estudada, no período de janeiro a dezembro de 2014. Os dados foram compilados de forma individual, ou seja, de acordo com a Unidade Operacional, permitindo a análise de cada batalhão e comparativa entre os mesmos.

\section{RESULTADOS}

As três Unidades do Comando de Policiamento do Interior Um investigadas têm um total de 1058 Policiais Militares, sendo 385 no $1^{\circ}$ BPM/I, 263 no $41^{\circ}$ BPM/l e 410 no $46^{\circ} \mathrm{BPM} / \mathrm{l}$.

Foram analisadas, de forma global, as doenças ortopédicas, psiquiátricas, cardiorrespiratórias, gastrointestinais, dermatológicas e renais que acometeram os Policiais Militares no ano de 2014.

Tabela 1 - Prevalência das doenças que acometeram os Policiais Militares dos BPMI no período de Janeiro a Dezembro de 2014.

\begin{tabular}{|c|c|c|c|c|c|c|c|c|c|c|c|c|c|}
\hline & & & & & & & & & & & & & Total \\
\hline & Jan & Fev & Mar & Abr & Mai & Jun & Jul & Ago & Set & Out & Nov & Dez & \\
\hline \multicolumn{14}{|l|}{$1^{\circ} \mathrm{BPM} / \mathrm{I}$} \\
\hline Ortopedia & 24 & 20 & 25 & 19 & 13 & 29 & 34 & 29 & 29 & 21 & 28 & 31 & \multirow{2}{*}{$\begin{array}{c}302 \\
86\end{array}$} \\
\hline Psiquiatria & 6 & 6 & 2 & 5 & 8 & 7 & 11 & 13 & 10 & 12 & 5 & 1 & \\
\hline Cardio/respiratório & 5 & 11 & 11 & 13 & 11 & 16 & 15 & 12 & 9 & 4 & 17 & 7 & 131 \\
\hline Gastro & 6 & 7 & 8 & 10 & 9 & 13 & 7 & 9 & 10 & 8 & 9 & 8 & 104 \\
\hline Dermato/renais & 9 & 10 & 10 & 10 & 4 & 4 & 7 & 5 & 7 & 8 & 2 & 1 & 77 \\
\hline Total & 50 & 54 & 56 & 57 & 45 & 69 & 74 & 68 & 65 & 53 & 61 & 48 & \\
\hline \multicolumn{14}{|l|}{$41^{\circ} \mathrm{BPM} / \mathrm{I}$} \\
\hline Ortopedia & 19 & 18 & 18 & 15 & 10 & 16 & 22 & 10 & 11 & 17 & 6 & 10 & 172 \\
\hline Psiquiatria & 8 & 7 & 5 & 11 & 9 & 9 & 11 & 5 & 8 & 9 & 6 & 9 & 97 \\
\hline Cardio/respiratório & 5 & 8 & 2 & 2 & 2 & 5 & 8 & 8 & 2 & 2 & 3 & 2 & 49 \\
\hline Gastro & 9 & 3 & 3 & 3 & 4 & 5 & 5 & 4 & 2 & 2 & 2 & 2 & 44 \\
\hline Dermato/renais & 3 & 4 & 2 & 0 & 0 & 3 & 1 & 7 & 9 & 5 & 3 & 7 & 44 \\
\hline Total & 44 & 40 & 30 & 31 & 25 & 38 & 47 & 34 & 32 & 35 & 20 & 30 & \\
\hline \multicolumn{14}{|l|}{$46^{\circ} \mathrm{BPM} / \mathrm{I}$} \\
\hline Ortopedia & 59 & 35 & 30 & 27 & 22 & 31 & 26 & 34 & 23 & 19 & 16 & 21 & 343 \\
\hline Psiquiatria & 3 & 13 & 14 & 10 & 16 & 10 & 11 & 10 & 8 & 6 & 7 & 3 & 111 \\
\hline Cardio/respiratório & 10 & 6 & 6 & 14 & 12 & 13 & 4 & 9 & 13 & 4 & 8 & 7 & 106 \\
\hline Gastro & 5 & 9 & 10 & 8 & 10 & 5 & 6 & 7 & 14 & 6 & 2 & 10 & 92 \\
\hline Dermato/renais & 3 & 5 & 4 & 5 & 3 & 4 & 5 & 8 & 3 & 8 & 6 & 5 & 59 \\
\hline Total & 80 & 68 & 64 & 64 & 63 & 63 & 52 & 68 & 61 & 43 & 39 & 46 & \\
\hline
\end{tabular}

Fonte: Autor. 
De acordo com a Tabela 1, nos três batalhões, as doenças tiveram uma distribuição regular ao longo do ano. Verifica-se que as doenças ortopédicas, cardiorrespiratórias e psiquiátricas obtiveram maiores índices, em todos os meses, nos Batalhões analisados. Ressalta-se que as doenças ortopédicas foram as que tiveram maior prevalência durante todo o ano nos três batalhões.

O Gráfico 3 apresentou semelhança com os gráficos 1 e 2, destacando-se a ocorrência preponderante das doenças ortopédicas (343), seguidas das doenças psiquiátricas (111), cardiorrespiratórias (106), doenças gastrointestinais (92) e dermatológicas ou renais (59).

\section{Gráfico 1 - Prevalência das doenças que acometeram os Policiais Militares do $1^{\circ}$ BPMI no período de Janeiro a Dezembro de 2014.}

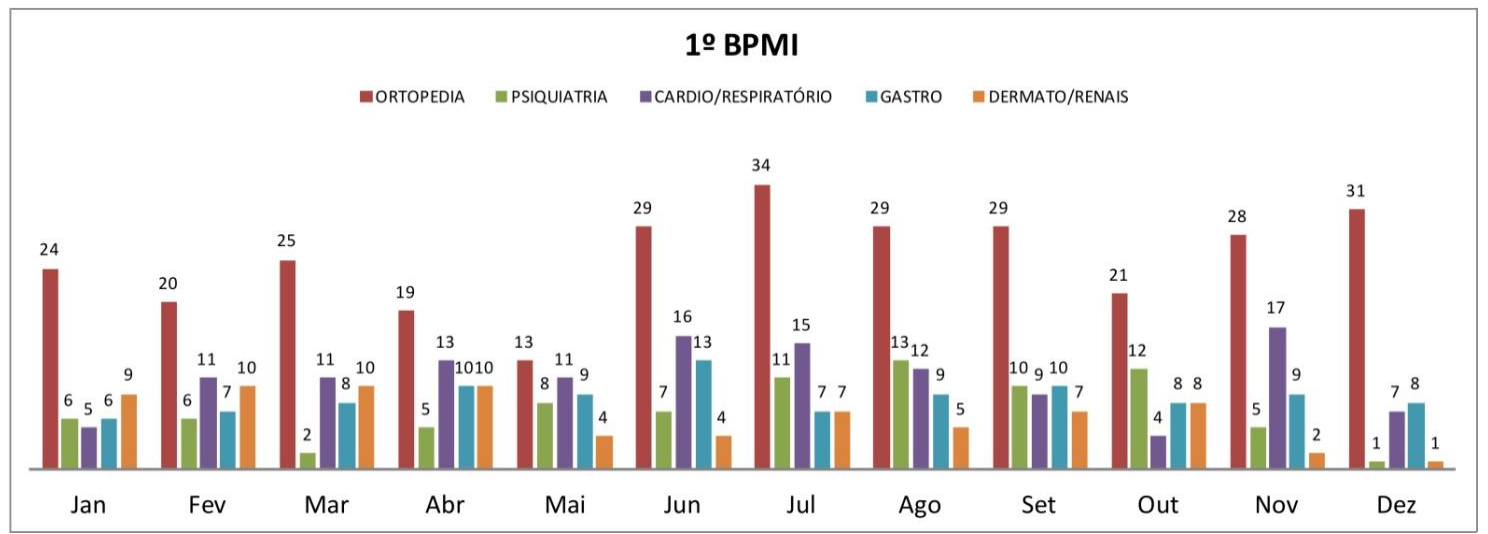

Fonte: Autor.

Segundo o Gráfico 1, as doenças apresentaram distribuição regular durante todo o ano. As doenças ortopédicas (302) prevaleceram, apresentando maior índice de acometimentos, seguidas de alterações cardiorrespiratórias (131) e psiquiátricas (86), no período estudado. As doenças gastrointestinais (104) prevaleceram entre os meses de abril e junho, e as dermatológicas/renais (77), nos meses de fevereiro, março e abril.

Gráfico 2 - Prevalência das doenças que acometeram os Policiais Militares do $41^{\circ}$ BPM no período de Janeiro a Dezembro de 2014.

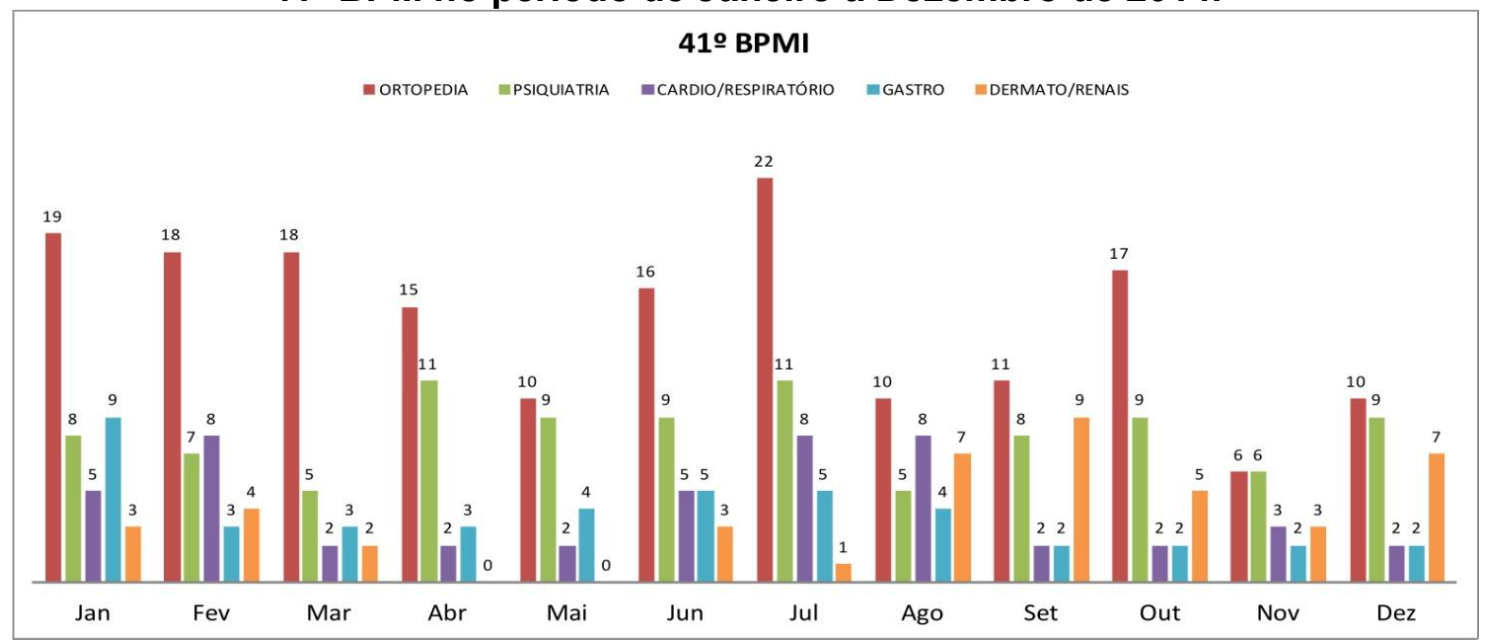

Fonte: Autor. 
De acordo com o Gráfico 2, as doenças no $41^{\circ}$ Batalhão também apresentaram distribuição regular. Doenças ortopédicas (172) foram prevalentes, seguidas das psiquiátricas (97) e cardiorrespiratórias (49). As doenças gastrointestinais (44) e dermatológicas ou renais (44) tiveram o mesmo número de ocorrências durante o ano.

\section{Gráfico 3 - Prevalência das doenças que acometeram os Policiais Militares do} 46 BPMI no período de Janeiro a Dezembro de 2014.

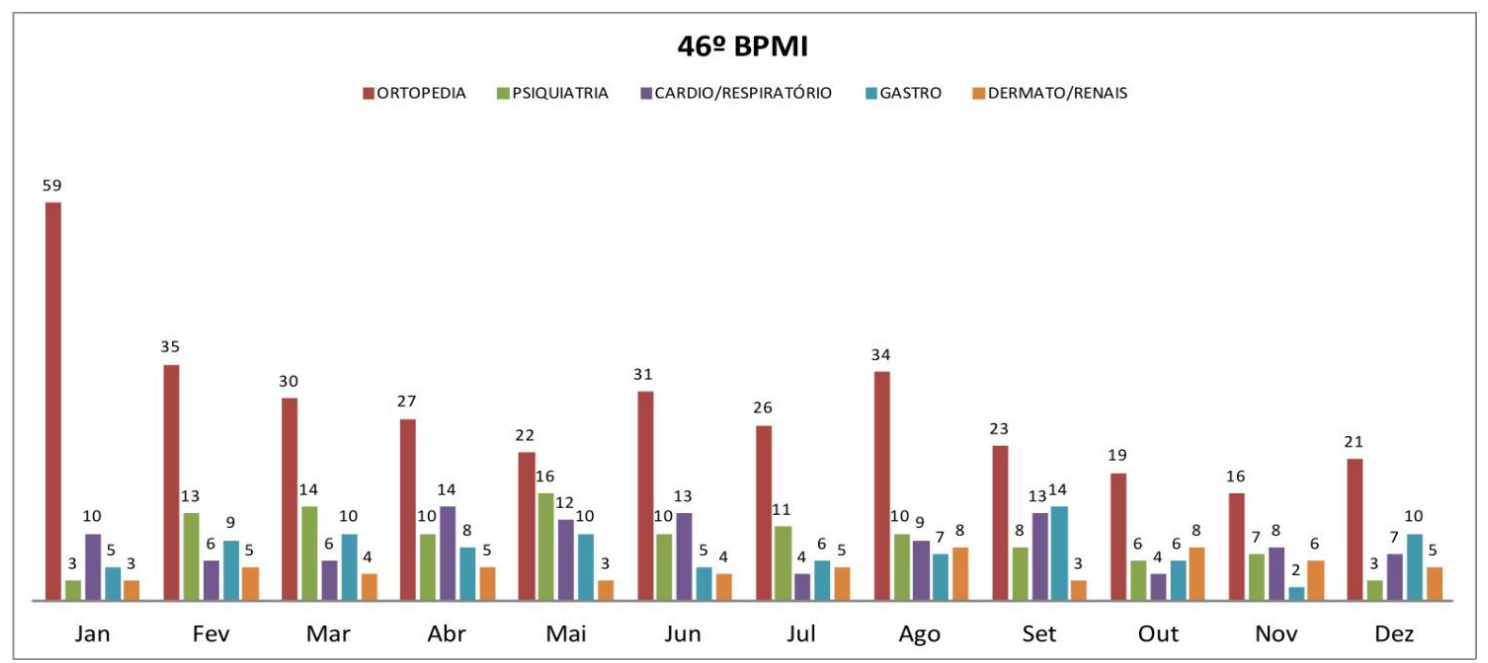

Fonte: Autor.

O Gráfico 4 mostra que 26 policiais militares dos batalhões estudados foram afastados de suas atividades, por Licença de Tratamento de Saúde, em virtude das doenças ortopédicas.

Gráfico 4 - Prevalência das doenças ortopédicas que acometeram os Policiais Militares, no período de Janeiro a Dezembro de 2014, que resultaram em período de Licença para Tratamento de Saúde (LTS).

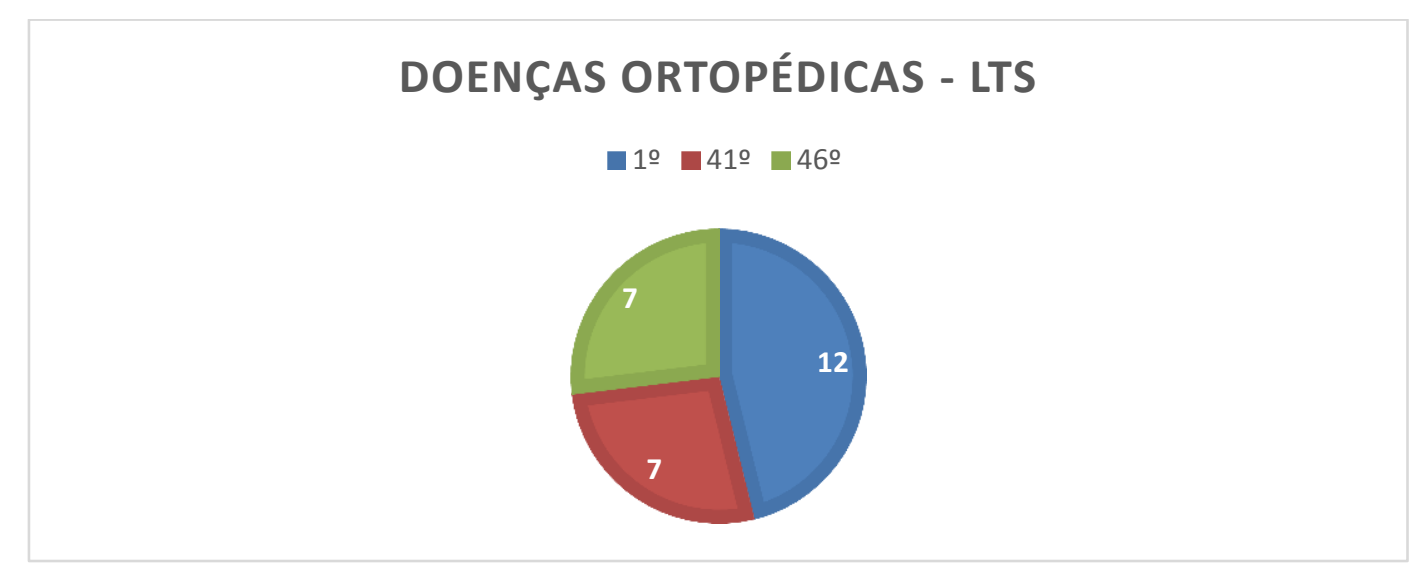

Fonte: Autor. 
O Gráfico 5 mostra que 11 policiais militares da amostra estudada foram afastados por Licença de Tratamento de Saúde em decorrência de doenças psiquiátricas.

\section{Gráfico 5 - Prevalência das doenças psiquiátricas que acometeram os Policiais Militares, no período de Janeiro a Dezembro de 2014, que resultaram em Licença para Tratamento de Saúde.}

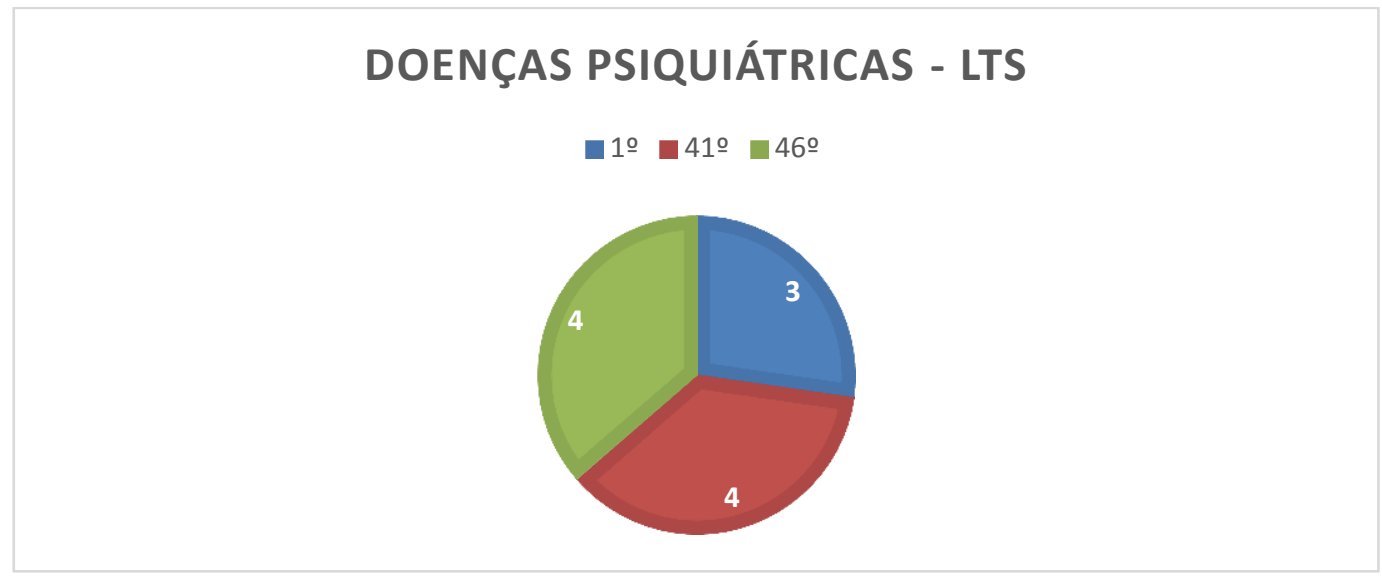

Fonte: Autor.

\section{DISCUSSÃO}

De acordo com os dados analisados nas três unidades dos batalhões, as doenças se assemelham em frequência, apresentando maior prevalência de doenças ortopédicas. Os resultados reforçam o estudo realizado por Pinto (2010), que expõe as posturas forçadas exigidas pelo exercício da profissão de Policial Militar. A causa dessas doenças pode ser a sobrecarga exercida pelos EPI's, bem como a força utilizada no desempenho de suas funções. É importante ressaltar que esses profissionais estão constantemente expostos ao estresse e à tensão muscular, em virtude do risco que correm durante sua jornada de trabalho. Os traumatismos, ferimentos, fraturas, luxação, entorses e distensão, são comuns a estes. As lesões e os quadros álgicos do policial militar estão relacionados com seus treinamentos táticos e físicos, pela complexidade de sua função, bem como ao uso de equipamentos táticos e de proteção individual (PAULO et al., 2014).

Os transtornos psíquicos podem estar relacionados à profissão de Policial Militar em virtude das peculiaridades da função exercida, pela exposição à violência urbana e mazelas sociais, vinculadas, ainda, a fatores como salário, alimentação inadequada e falta de exercícios físicos. Supõe-se, também, que o exercício profissional gere importante desgaste físico e psicológico, ocasionados por turnos de trabalho exigentes e excessivos, por períodos que chegam a 24 horas, com turnos de 12 horas, 8 horas ou 6 horas. Em muitas ocasiões, os Policiais Militares ultrapassam esses horários, sob permanente tensão e estresse, trabalhando com EPIs desconfortáveis e pesados 
(PINTO, 2010).

No bojo da profissão de policial, existem peculiaridades específicas que contribuem para o adoecimento, entre elas, a dedicação integral ao trabalho, o que leva a atividade profissional a sobrepor espaços de sua vida pessoal. Como consequência, desenvolve-se a necessidade de estar sempre atento diante de qualquer indivíduo e ambiente desconhecido, diminuindo suas relações sociais. Somatizam, ainda, o contato do policial com a criminalidade e a violência (CASTRO; CRUZ, 2015).

As doenças cardiorrespiratórias apresentaram distribuição regular, apresentando um discreto aumento de incidência nos meses de Junho e Julho, que pode ser associado à chegada do inverno, estação do ano caracterizada por queda na temperatura e associada a doenças que acometem esse aparelho.

As doenças gastrointestinais apresentaram prevalência nos meses de maio, junho e setembro. Aparentemente, estão associadas à alimentação inadequada do policial militar, que, muitas vezes, recorre a lanches rápidos durante seu turno de trabalho. Ressalta-se, ainda, que a própria atividade do policial pode contribuir para o desenvolvimento de doenças digestivas, tais como gastrite e úlcera, devido ao estresse com o qual muitos convivem durante a jornada de trabalho.

Sugere-se que as doenças dermatológicas estejam associadas ao uso contínuo do colete balístico, que causa grande desconforto térmico e atrito com a pele, bem como o contato direto do policial militar com outros indivíduos. Por sua vez, as doenças renais podem estar associadas diretamente à hipertensão arterial e pouca ingestão de água durante a jornada de trabalho (VASCONCELOS, 2007).

Dados do Instituto Brasileiro de Geografia e Estatística (IBGE), em 2013, informam que o país possui um policial militar para cada 473 habitantes e, especificamente, no estado de São Paulo, um policial militar para 488 habitantes (INSTITUTO..., 2015).

Considerando o exposto e fazendo uma análise crítica, in tese, a cada policial militar do estado de São Paulo afastado por licença para tratamento de saúde, 488 habitantes deixarão de ter a "Sensação de Segurança" e os serviços prestados por esse profissional.

\section{CONCLUSÃO}

O presente estudo permitiu traçar um retrato da prevalência de doenças que acometeram os policiais militares de um grande comando policial do interior do estado de São Paulo, no período de Janeiro a Dezembro de 2014. Foram prevalentes as doenças ortopédicas, psiquiátricas e cardiorrespiratórias, nessa ordem. Acredita-se que todas estejam associadas às características das atividades desenvolvidas pelo 
policial militar durante sua jornada de trabalho, por ser uma profissão que expõe, constantemente, o profissional a pesados equipamentos de proteção e ao risco de morte.

\section{REFERÊNCIAS}

CASTRO, M. C. D.; CRUZ, R. M. Prevalência de Transtornos Mentais e Percepção de Suporte Familiar em Policiais Civis. Psicol. Cienc. Prof., v. 35, n. 2, 2015.

FERREIRA, D. K. S., BONFIM, C., AUGUSTO, L. G. S. Fatores Associados ao Estilo de Vida de Policiais Militares. Rev. Ciência \& Saúde Coletiva, v. 6, n. 8, p. 3403-12, 2011.

GONÇALVES, S. J. C; VEIGA, A. J. S; RODRIGUES, L. M. S. Qualidade de vida dos policiais militares que atuam na área da $2^{\mathrm{a}} \mathrm{CIA}$ do $10^{\circ}$ Batalhão Militar (Miguel Pereira e Paty do Alferes). Revista Fluminense de Extensão Universitária, v. 2, n. 2, p. 5376, 2012.

INSTITUTO BRASILEIRO DE GEOGRAFIA E ESTATISTICA - IBGE. Pesquisa de informações Básicas Estaduais e Municipais. Perfil dos Estados e dos Municípios Brasileiros, Rio de Janeiro: IBGE, 2015. 80p. Disponível em <http://www.ibge.gov.br/biblioteca/visualizacaoo/livros/liv94541.pdf>. Acesso em: 20 ago. 2015

PAULO, L. F. L. et al. Prevalência de dor em alunos da academia de polícia militar do Barro Branco. Revista Delfos, n. 4, p. 5-11, 2014.

PINTO, J. N. Absenteísmo por Doença na Brigada Militar de Porto Alegre, RS. Trabalho de Conclusão de Curso (Graduação em Enfermagem) - Escola de Enfermagem da Universidade Federal do Rio Grande do Sul, Porto Alegre, 2010.

SILVA, R, SCHICHTING A. M. Aspectos relacionados à qualidade de vida e atividade física de policiais militares de Santa Catarina - Brasil. Revista Motricidade, v. 8, n. 3, p. 81-89, 2012.

SILVA, F. C et al. Qualidade de vida de policiais: uma revisão sistemática de estudos observacionais. Rev. Cubana de Medicina Militar, v. 43, n. 3, p. 341-51, 2014.

SIMÕES, M. C. Formulação de um repositor hidroeletrolítico para o trabalho físico ostensivo de policiais militares, adaptado as variações climáticas de Florianópolis. Florianópolis, 2003. 271f. Tese (Doutorado em Engenharia de Produção) - Universidade Federal de Santa Catarina. Florianópolis, 2003.

VASCONCELOS, I. C. Estudo ergonômico do colete à prova de balas utilizado na atividade policial. Bauru, 2007. 101f. Dissertação (Mestrado em Desenho Industrial) Faculdade de Arquitetura, Artes e Comunicação da Universidade Estadual Paulista "Júlio de Mesquita Filho", Bauru, 2007. 